\title{
Arvejas Obonuco Andina y Obonuco San Isidro como alternativas productivas para la sustitución de cultivos ilícitos en el sur de Colombia
}

\section{Green beans Obonuco Andina and Obonuco San Isidro as productive alternatives for the substitution of illicit crops in the south of Colombia}

María Victoria Zuluaga-Mogollón'
María del Pilar Zuluaga-Mogollón
Yanine Rozo-Leguizamón

Recibido: marzo 17 de 2019

Aceptado: junio 28 de 2019

\section{Resumen}

El objetivo de esta investigación retrospectiva de tipo cualitativo, fue develar los efectos percibidos por los productores de arveja del municipio de Puerres, ubicado en el departamento de Nariño (Colombia), tras la adopción de las variedades de arveja (Pisum sativum) Obonuco Andina y Obonuco San Isidro. Para esto, se adelantó un proceso de construcción participativa con población rural campesina e indígena, con quienes fueron analizadas las dinámicas productivas, socioculturales y ambientales del territorio. El estudio abarcó el período comprendido entre 1980 y 2017, determinando la percepción de los dos grupos focales, frente a las dimensiones mencionadas y sus efectos en el territorio. Los resultados indican que, además de los atributos agronómicos de las dos variedades objeto de estudio y de su aceptación en el mercado, se presentaron aspectos que presionaron el territorio y que actuaron como catalizadores para su adopción. Igualmente, se determinó que, además de la reconversión productiva de cultivos de cereales, estas variedades se establecieron como la opción productiva tanto económi-

\begin{abstract}
The objective of this retrospective qualitative research was to reveal the effects perceived by peas producers in the municipality of Puerres located in the Department of Nariño (Colombia), after the adoption of varieties of pea (Pisum sativum) Obonuco Andina and Obonuco San Isidro. For this reason, a process of participatory construction was carried out with rural and indigenous population, with whom the productive, sociocultural and environmental dynamics of the territory were analyzed. The study covered the period between 1980 and 2017 which allowed us to determine the perception of the two focus groups against the mentioned dimensions and their effects on the territory. The results indicate that, in addition to the agronomic attributes of the two varieties under study and their acceptance in the market, aspects were presented that pressed the territory and acted as catalysts for its adoption. Likewise, it was determined that, in addition to the productive reconversion of cereal crops, these varieties were established as the productive option both economically and socially sustainable, given the need
\end{abstract}

1 Ingeniera Agrónoma, Estudiante de Maestría en Desarrollo Rural, Corporación Colombiana de Investigación Agropecuaria (AGROSAVIA), Mosquera, Colombia. E-mail: mzuluaga@agrosavia.co ORCID: 0000-0002-3246-3400

2 Filósofa, Magíster en Historia, Universidad de Bogotá Jorge Tadeo Lozano, Bogotá, Colombia. E-mail: maria.zuluaga@utadeo.edu.co ORCID: 0000-0001-7757-8116

3 Ingeniera Agrónoma, Magíster en Planificación Social, Corporación Colombiana de Investigación Agropecuaria (AGROSAVIA), Cúcuta, Colombia. E-mail: yrozo@agrosavia.co ORCID: 0000-0003-4723-478 
ca como socialmente sostenible, ante la necesidad de la sustitución de cultivos ilícitos en el sur de Colombia.

Palabras clave: pisum sativum, territorio, adopción de tecnología, desarrollo rural, evaluación de tecnologías.

\section{Introducción}

La Organización para la Cooperación y el DesarroIlo Económico, OCDE (2013), indica que: "el sistema de innovación de Colombia es aún pequeño, excesivamente centrado en actores estatales y su desempeño se encuentra muy por debajo del nivel requerido para su desarrollo sostenido". La innovación está relacionada con la adopción de tecnología, la que a su vez se encuentra condicionada por diversos factores que trascienden lo técnico y lo económico (Molina, 2009; García-Parra \& Plazas-Leguizamón, 2019).

La adopción de tecnología se encuentra condicionada por los aspectos espacio-temporales de un territorio, que responde a los desafíos del momento histórico, como son los cambios inducidos por el proceso de globalización (Vásquez, 2007). Tal es el caso de la reforma económica de los años noventa, generada a nivel mundial, que impactó en lo glocal. En este caso, cultivos que contaban con precios de sustentación e intervención, fueron los que sufrieron el mayor impacto (CEPAL, 2000).

En Colombia, según la Federación Nacional de Cultivadores de Cereales y Leguminosas, FENALCE (sf), la siembra de cereales pasó de 1.1 millones de hectáreas en el año 1989, a 588.499 diez años más tarde. La gran disminución de las áreas de siembra y cosecha de trigo, cebada, frijol y maíz, reporta- for the substitution of illegal crops in southern Colombia.

Keywords: pisum sativum, territory, tecnology adoption, rural development, technologies assesment.

das a nivel nacional, coincidió con su disminución en el departamento de Nariño, Colombia. Esto generó desafíos productivos, sociales y económicos en varios municipios cerealistas del sur de Nariño (Castillo-Burbano \& Jurado, 2014).

Con el fin de brindar alternativas productivas para el desarrollo del territorio, entidades como: PRONATTA, FENALCE y CORPOCEBADA, destinaron recursos para trabajar en la cadena agroalimentaria de arveja en el departamento de Nariño (CORPOICA, S.F.) financiando proyectos desarrollados por la Universidad de Nariño y la Corporación Colombiana de Investigación Agropecuaria, Corpoica, hoy AGROSAVIA. Hacia el primer quinquenio del siglo XXI, son liberados los materiales de arveja Obonuco Andina y Obonuco San Isidro, orientados a mejorar el sistema de economía campesina en municipios de la Provincia de Obando, en el sur de Nariño (FENALCE, S.F.). Desde el lanzamiento de las dos variedades de arveja, se evidencia el aumento en las áreas de siembra y de cosecha del departamento, pasando de un área sembrada inferior a las 2000 hectáreas a principios de la década de los años 90, a 5000 hectáreas a finales de la misma década y un área sembrada cercana a las 15.000 hectáreas para el año 2013.

Las variedades de arveja Obonuco Andina y San Isidro, fueron generadas en respuesta a la caída en los precios, principalmente de trigo y cebada, 
ocasionada por la apertura económica de los años 90. El aumento en el área cultivada en arveja ha derivado en que Nariño ostente en la actualidad el $52 \%$ de la producción nacional (Ministerio de Agricultura y Desarrollo Rural, 2014). Esto ha sido atribuido a la productividad de estas dos variedades, al igual que a la calidad de la vaina y de su grano, lo que ha permitido su comercialización fuera del departamento, llegando incluso al interior del país (Zuluaga-Mogollón, 2019).

El contexto histórico que enmarcó la adopción de las dos variedades de arveja mencionadas, así como su posicionamiento, por ya casi 15 años desde su lanzamiento y su efecto en las dinámicas del municipio de Puerres, Nariño, lo constituyen en un caso de estudio para entender las dinámicas del territorio. En este sentido, el objetivo de esta investigación fue develar los efectos percibidos por los productores de arveja del municipio de Puerres, a través de un proceso de construcción participativa con población rural campesina e indígena, con quienes fueron analizadas las dinámicas productivas, socioculturales y ambientales del territorio.

\section{Materiales y métodos}

Con el fin de propiciar el proceso de construcción participativa, el cual requiere de trabajo transdisciplinar, en el presente estudio se empleó como herramienta el Diagnóstico Rural Participativo, DRP. Se contó con la confluencia de diversos actores, el reconocimiento directo del territorio y como base teórica el análisis de documentación existente (Valderrama-Hernández, 2013). El tipo de muestreo adoptado fue no probabilístico intencional, donde se eligieron casos que representan a la población o universo (Silva \& Menezes, 2005). Se empleó un diseño de tipo exploratorio-descriptivo, no experimental, de corte transversal en grupos focales, según Martínez-Miguélez (2004).

En el municipio de Puerres se cuenta con mayoría compartida de población indígena y campesina, por lo que se constituyeron dos grupos focales de discusión. El primero conformado por la población indígena perteneciente al Cabildo Indígena El Gran Tescual, y un segundo grupo conformado por población campesina de la Asociación Nacional de Usuarios Campesinos de Colombia, ANUC, en el municipio de Puerres, departamento de Nariño, Colombia.

Las dinámicas en el territorio y la incidencia en la adopción de las arvejas Obonuco Andina y Obonuco San Isidro, se determinaron a partir de Talleres Participativos, TP, adelantados en enero de 2018. El desarrollo de los TP se orientó hacia la pluralidad de experiencias en las que, tras la complementariedad, se analizaron variables productivas, ambientales, socioculturales y políticas, en los últimos 30 años. Según la metodología propuesta por Gelfius (2002), a partir de la información obtenida, junto con los participantes en los talleres, se construyeron diagramas de flujo espacio-temporal del municipio, entre la década de los años 80 y la actualidad. Además, se elaboraron mapas históricos de los recursos y uso de la tierra.

\section{Resultados y discusión}

En esta sección, los apartados entre comillas, salvo que se indique lo contrario, corresponden a información sin editar, extraída de los talleres de DRP en Puerres, adelantados en enero de 2018.

\section{Línea espacio-temporal}

Dimensión productiva. En los TP, se evidenció que antes de la década de los años 70, el intercambio y el trueque de productos cultivados en las diferentes alturas e incluso entre vecinos, eran la base del sistema productivo y económico del municipio. En la década de los 80 , se intensifica la producción con la incorporación de agroquímicos, con lo que, tanto campesinos como indígenas perciben el incremento en la rentabilidad de los cultivos. En la década de los 90 , con el aumento en las plagas, se acentúa la utilización de agroquímicos en los cultivos y con ello la llegada de multinacionales a la región. 
“En los años 90 el 100\% de los terrenos tenían arveja...con la liberación de la arveja Sindamanoy en el 95, se genera gran impacto productivo y social... De Puerres salían 6 turbos por semana, transportando la arveja".

A comienzos del siglo XXI, con el propósito de mejorar la calidad fitosanitaria de la arveja, así como la producción por unidad de área, se pasa de un sistema de siembra rastrero sin soporte de plantas, en el que las pérdidas por incidencia de insectos plaga y enfermedades eran considerables, a la utilización de un sistema de tutorado con espaldera y enmallado. Con esta medida se logró el incremento del $15 \%$ en el rendimiento de la variedad de arveja ICA-CORPOICA Sindamanoy (ICA-CORPOICA, 1995). Con la introducción de las variedades Obonuco Andina y Obonuco San Isidro, el rendimiento en este cultivo se incrementó en 36 y 16\%, con respecto a la variedad predecesora ICA-CORPOICA Sindamanoy (CORPOICA - PRONATTA, 2002).

Con la tecnificación del cultivo de arveja, además de lograr el incremento en la producción y mejorar la calidad del producto, se logró la producción de arveja fresca a lo largo de todo el año, permitiendo el posicionamiento de la arveja de Puerres en el mercado nacional. En la actualidad, el departamento de Nariño ostenta el primer puesto como productor de arveja en vaina verde del país y se constituye en fuente de ingresos y generador de empleo para la región, haciendo de este un sistema social y económicamente sostenible por cerca de 15 años (Zuluaga-Mogollón, 2019).

En el siglo XXI, la discusión cobra un interés particular, principalmente en el grupo del campesina- do, quienes ponen de manifiesto acontecimientos que marcaron el destino del municipio:

"Se dejan de cultivar alimentos por el auge de la amapola: era más rentable... la producción de cultivos lícitos para el 2003 se baja y aumentan los ingresos por los cultivos ilícitos y con esto se aumenta en la remuneración de los jornales... la rentabilidad era del 300 por ciento con respecto a otros cultivos.... Se generaron otras fuentes de ingresos, como tiendas, graneros, cantinas, bares y prostíbulos, había derroche... comienza el comercio de las tierras por venta o arrendamiento... a mediados de la década, la arveja "evoluciona" y la amapola se acabó".

A lo largo de los más de 30 años analizados, se tiene la percepción de pérdida marcada de diversidad en la producción agrícola del municipio. A partir de los años 90, se pasa de una gran diversidad de productos cultivados, como: maíz, cebada, trigo, quinua, ocas, hoyocos, arracacha, haba, papa (criolla), frijol, calabaza, repollo, zapallos, cebollas y fique magüa, entre otros, a unas cuantas especies cultivadas en las últimas dos décadas. Lo anterior se refleja en la tabla 1, elaborada a partir de la línea de tiempo, construida con los participantes en la discusión de los grupos focales.

En la primera década del siglo XXI, se acentúa la pérdida de diversidad, evidenciada por la disminución en la variedad de productos cultivados, principalmente por parte del campesinado. Estos se concentraron en la producción de: papa (Morasurco, Pastusa, Mambera, ICA - Huila), arveja (Sindamanoy y Sta Isabel), quinua y frijol (Cargamanto y Bolón rojo), ver tabla 1. 
Tabla 1. Dinámica productiva de Puerres entre 1980 y 2017.

\begin{tabular}{|c|c|c|c|c|c|c|c|}
\hline \multicolumn{2}{|c|}{ 1980-1989 } & \multicolumn{2}{|c|}{ 1990-1999 } & \multicolumn{2}{|c|}{ 2000-2009 } & \multicolumn{2}{|c|}{$2010-2017$} \\
\hline Indígenas & Campesinos & Indígenas & Campesinos & Indígenas & Campesinos & Indígenas & Campesinos \\
\hline Cebada & Cebada & $\begin{array}{c}\text { Papa (Criolla) } \\
\text { Calabaza }\end{array}$ & $\begin{array}{c}\text { Papa (Morasurco, } \\
\text { Pastusa, Mambera, } \\
\text { ICA-Huila) }\end{array}$ & $\begin{array}{l}\text { Arveja (Sinda- } \\
\text { manoy, San } \\
\text { Isidro, Andina) }\end{array}$ & $\begin{array}{c}\text { Arveja (Sinda- } \\
\text { manoy, San } \\
\text { Jorge, } \\
\text { Piquin egra) }\end{array}$ & $\begin{array}{l}\text { Arveja (San } \\
\text { Isidro, Andina, } \\
\text { Piquinegra } \\
\text { var.) }\end{array}$ & $\begin{array}{l}\text { Arveja (Andi- } \\
\text { na, San Isidro, } \\
\text { Sureña) }\end{array}$ \\
\hline Trigo & Maíz & Frijol & $\begin{array}{c}\text { Arveja } \\
\text { Sindamanoy * } \\
\begin{array}{c}\text { Sta Isabel=Piquine- } \\
\text { gra* }\end{array}\end{array}$ & Frijol & Amapola & $\begin{array}{c}\text { Papa (Criolla, } \\
\text { Mambera, } \\
\text { Ratona) }\end{array}$ & $\begin{array}{c}\text { Frutales } \\
\text { (mora, tomate } \\
\text { de árbol, } \\
\text { fresa, curuba, } \\
\text { uchuva) }\end{array}$ \\
\hline Quinua & $\begin{array}{l}\text { Frijol (cho- } \\
\text { cho) }\end{array}$ & $\begin{array}{c}\text { Arveja (Pi- } \\
\text { quinegra, Sta } \\
\text { Isabel) }\end{array}$ & $\begin{array}{c}\text { *en el } 100 \% \text { de los }^{\text {terrenos }}\end{array}$ & Zanahoria & $\begin{array}{c}\text { Arveja (Andi- } \\
\text { na, San Isidro) }\end{array}$ & $\begin{array}{l}\text { Hortalizas } \\
\text { (lechuga, } \\
\text { calabazín) }\end{array}$ & $\begin{array}{l}\text { Papa (Parda, } \\
\text { Supremas, } \\
\text { criolla, Ocari- }\end{array}$ \\
\hline Ocas & Quinua & Cebolla & Quinua & Mora & Papa (Capiro y & & na, yema de \\
\hline Hoyocos & Oca & Repollo & & & Pastusa) & Frutales & huevo) \\
\hline Arracacha & Arracacha & Lechuga & $\begin{array}{l}\text { Frijol (cargamanto, } \\
\text { bolón rojo) }\end{array}$ & $\begin{array}{l}\text { Tomate de } \\
\text { árbol }\end{array}$ & Hortalizas & $\begin{array}{l}\text { (Uchuva, } \\
\text { fresa) }\end{array}$ & Leche \\
\hline Chocha & Calabaza & Zanahoria & & Uchuva & Cuyes & Cuyes & Cuyes \\
\hline Habas & $\begin{array}{l}\text { Papa (Guata, } \\
\text { Diacol) }\end{array}$ & Arracacha & & Curuba & & $\begin{array}{l}\text { Ganadería - } \\
\text { lechería }\end{array}$ & \\
\hline Papa & $\begin{array}{l}\text { Arveja (Go- } \\
\text { rriona) }\end{array}$ & Maíz & & Amapola & & & \\
\hline Frijol & Zapayo & Trigo & & Ganadería - & & & \\
\hline Calabaza & Hoyocos & Cebada & & Lechería & & & \\
\hline Repollo & Habas & Zapallo & & Cuyes & & & \\
\hline $\begin{array}{l}\text { Zapallo } \\
\text { Cebollas } \\
\text { Fique }\end{array}$ & $\begin{array}{c}\text { Maguas (Para } \\
\text { trampas } \\
\text { de gusano } \\
\text { blanco) }\end{array}$ & Cuyes & & & & & \\
\hline
\end{tabular}

En el período evaluado en la línea de tiempo, se evidencia que la pérdida de diversidad estuvo relacionada con el impacto que ejerció el cultivo de amapola en el territorio, principalmente entre los años 2003 y 2006. No obstante, desde principios de la década de los 90 , por contar con las condiciones aptas para la producción de amapola, se había generado la alerta para los municipios del sur de Nariño, como: El Tablón, Cumbitara, Santacruz, Túquerres, Sandoná, Puerres, San Lorenzo, Contadero, Córdoba, Iles, Leiva, Ipiales y La Cruz (El Tiempo, 1992).

Además de ocasionar la pérdida de diversidad agrícola, el auge de la amapola lleva a la amplia- ción de la frontera agrícola hacia zonas de páramo, las que hasta ese momento habían servido de reserva. Este aspecto se menciona por pobladores de la región:

"Se dejan de cultivar alimentos por el auge de la amapola: era más rentable... a mediados de la década, la arveja "evoluciona" y la amapola se acabó".

"Los cultivos ilícitos que hubo en esta región fueron en si desde el 2003, por ahí empezaron a producir, pero fue arto lo que se sembró.... Pues aquí en el frío da la amapola, pero eso ya se quitó... póngale 2006 , se había acabado ya totalmente, y 
siguieron sembrando arveja" (R. Güepud, comunicación personal, octubre 26 de 2017).

Con la llegada al territorio del programa de erradicación de cultivos ilícitos en 2007, con el que se implementan medidas sancionatorias a los cultivadores de amapola, se genera la necesidad de optar por alternativas productivas económicamente estables, intensificando el cultivo de arveja, para entonces, con la adopción de las nuevas variedades de arveja Obonuco Andina y Obonuco San Isidro.

“Para el año 2010, la región...cuenta con centro de acopio para la comercialización de arveja en Ipiales, de donde salen entre 10 y 15 camiones diariamente hacia el resto del país... esto hace que Puerres sea reconocido a nivel nacional".

En este momento, la línea de tiempo evidencia por parte de indígenas y campesinos, la reducción en la producción del cultivo, pasando de un promedio entre 8 y 10 bultos, 10 años atrás, a una producción entre 6 y 8 bultos por kilo de semilla en la actualidad, con alta fluctuación en los precios. Según los participantes en el taller, para el año 2017 el precio de la arveja estuvo entre $\$ 300.000 /$ bulto (máximo) y \$20.000/bulto (mínimo), además del aumento en los costos de producción, lo que hace que la rentabilidad del cultivo disminuya. Dada la sobreoferta de arveja y la inestabilidad en el precio, en la actualidad se está incursionando en otros cultivos, principalmente frutales, como: mora, tomate de árbol, fresa, curuba y uchuva, ver tabla 1.

Dimensión ambiental. A finales de la década de los años 60 llega a la región el Oleoducto Trasandino - OTA que, si bien, no se tiene la percepción por parte de los participantes en los talleres de la generación de beneficios económicos, ni sociales para el municipio de Puerres, si se evidenció la incidencia que este tuvo en aspectos ambientales.

En la década de los 80 , con la colonización de tierras de reserva, las cuales fueron tituladas por el Instituto Colombiano de la Reforma Agraria, INCO-
RA, se acentúa la tala de bosques para la ampliación de la frontera agrícola, conllevando cambios en el destino del uso de la tierra. Esto, junto con la implementación del OTA, ha sido considerado por la población, como acontecimientos que afectaron considerablemente las reservas y fuentes de agua para la región. Ellos indican: "Para entonces, comienza a evidenciarse disminución en el suministro del agua, y el nivel freático del suelo es bajo".

De acuerdo con la percepción de los participantes en los talleres, en la década de los 90: “...se sintió el efecto del cambio climático y comenzaron los problemas de agua en el verano... episodios de Niño y Niña...se comienzan a presentar heladas... se vieron pequeños cambios en el paisaje". En este momento se evidencia el aumento en los insectos plaga y enfermedades, aumentando la utilización de agroquímicos, lo que incide, según los participantes, en la contaminación del agua y del medio ambiente. Esto se une a la mala disposición de los residuos, lo que persiste en la actualidad.

A comienzos del siglo XXI, se intensifica la producción agropecuaria con la introducción de la labranza mecanizada, lo que hasta el momento se hacía de manera tradicional con la labranza de bueyes.

La tecnificación de la producción agrícola, que en el cultivo de arveja se visibilizó con la implementación del sistema de tutorado de las plantas, con espaldera y enmallado, trajo consigo beneficios sociales y económicos para el municipio. No obstante, para la implementación del sistema fue necesaria la utilización de postes y varas de madera, con lo que se propició la tala de bosques nativos. Con el propósito de disminuir el impacto ocasionado, se promovió la siembra de especies arbóreas foráneas de rápido crecimiento, las que, de acuerdo con la percepción de entrevistados y participantes en talleres, afectaron la biodiversidad de la región, así como la fertilidad de los suelos.

La situación anterior se prolonga hasta la actualidad, y a juicio de los pobladores, ha incidido en el aumento de los eventos extremos de sequía y 
Iluvia. Estos aspectos productivos, sumados a actividades como la minería y la extracción de piedra, favorecieron la erosión de los suelos, contribuyendo al deterioro o agotamiento de los recursos del territorio.

Dimensión Sociocultural. Antes de la década de los años 70, la educación en la región era hasta los primeros años de escolaridad, máximo hasta tercer grado de primaria y se concentraba en la población masculina, lo que conduce a que un alto índice de la población mayor tenga escaso nivel de educación, incluso en la actualidad.

Lo anterior coincide con resultados obtenidos en el estudio de adopción de estas variedades de arveja, en los municipios de Córdoba y Puerres, en el que de 117 productores encuestados el 30\% cuentan con primaria completa y $44 \%$ primaria incompleta (Zuluaga-Mogollón, 2019). Esto contrasta con el informe de caracterización social y económica de Nariño, donde se menciona que el departamento presenta una cobertura en educación básica primaria del $75.78 \%$. En el caso de educación secundaria, la mayor cobertura, 70\%, la ostenta la Provincia de Obando, en donde se encuentra ubicado el municipio de Puerres (Castillo-Burbano \& Jurado, 2014).

En cuanto a aspectos culturales, antes de la década de los años 80 , se mantenían prácticas ancestrales de los pueblos indígenas de los Andes, como lo era el trabajo colectivo representado en las mingas para arreglo de caminos, en los que se ofrecían de manera voluntaria convites entre los mismos miembros de la comunidad y se adelantaban acciones para un bien común. Así mismo, con el fin de garantizar la alimentación y subsistencia de la familia indígena, se contaba con chagras. Las fiestas religiosas y patronales se organizaban a nivel de vereda, aplicaban la medicina natural y había hermandad.

Para la década de los 90, conforme con la percepción de los participantes en los talleres, hay pérdida de identidad cultural, pérdida de unión familiar por la tecnología, se aplica la medicina alópata y hay disminución de las chagras. "La participación en las mingas para arreglo de caminos era obligatoria, ya no era voluntaria".

En los años 80, la propiedad de la tierra se constituyó en un punto de inflexión en el municipio, al ser un tema generador de conflicto en la comunidad, mencionando que: "se presentó división de la comunidad por colonización de territorios sagrados, esto debido a la titulación de tierras a los colonos por parte del INCORA, lo que generó migración desde otros departamentos...."

Para finales de los años 90 , se pone de manifiesto la llegada al territorio de varios frentes de las FARC, situación que se evidencia a nivel nacional en 1997, con la masacre de 31 militares en Puerres, situación de violencia que se extiende a Córdoba, Potosí e Ipiales. Dicha situación, generó el desplazamiento de jóvenes del municipio a trabajar en el Putumayo.

A comienzos de siglo se presenta el auge de la amapola en el territorio (2003-2006), lo que tiene un efecto considerable en varias dimensiones de este:

"se presenta descomposición social, violencia, masacres, desplazamiento, pérdida de cultivos para la alimentación por siembra de amapola, actividad de la guerrilla y relación de esta con los cultivos ilícitos. En general, familias e incluso niños trabajaban en cultivos de amapola, por remuneración elevada. Hay migración desde otros departamentos (Valle, La Guajira, Huila, Antioqueños)...después de dejar la amapola aumenta el desempleo".

Luego de la erradicación de los cultivos de amapola y con el retorno a las actividades lícitas en el municipio, se pone de manifiesto por parte de los participantes en los talleres, la percepción de seguridad del territorio. La primera década del siglo XXI finaliza con una población que en su mayoría son oriundos del mismo municipio, lo que a su juicio, ha facilitado la organización comunitaria. 
Para el caso de la población indígena, se constituyó en Cabildo indígena El Gran Tescual y parte del campesinado que se unió a la ANUC.

Dada la reconfiguración en la estructura del tejido social del municipio, tras los acontecimientos que constriñeron el territorio en las décadas predecesoras, los participantes en los talleres manifestaron la pérdida marcada de la identidad cultural y pérdida de la unión familiar, acentuada con la llegada al municipio de las Tecnologías de la Información y la Comunicación, TIC. Esto relacionado, principalmente, con la ubicación de las antenas de varios operadores de telefonía en el municipio, que permiten el acceso a señal de celular e internet a buena parte de la población local y circundante. Igualmente, se hace referencia a la pérdida de costumbres ancestrales como las mingas para arreglo de caminos. Los carnavales, que anteriormente se realizaban en las veredas, en la actualidad son organizados en el casco urbano, con la incorporación de danzas, comparsas y murgas.

Pese a la actual percepción de ser un territorio seguro, este no se percibe por la población joven como un territorio con oportunidades para el buen vivir. Esto ha llevado a que la población de este grupo etario migre hacia otros departamentos para adelantar sus estudios, con un bajo nivel de retorno a su municipio. Esta situación es común a otros municipios colombianos, donde se genera un envejecimiento de los pobladores dedicados a las labores del campo (Casas-Herrera, Gil-León \& Forero-Medina, 2018).

Dimensión Política. La década de los 70, se caracterizó por la colonización de buena parte de las "tierras sagradas" (denominación otorgada por la población indígena), las que fueron adjudicadas por el INCORA durante la década de los 80 . Tras el impacto de la apertura económica de finales de los años 90, se dejan de cultivar productos como el trigo, la cebada y el maíz, con lo que se evidenció la desarticulación de las políticas públicas, favoreciendo la división política de la región.
Pese a la percepción de abandono del Estado, en el presente estudio se tienen hallazgos de acciones del nivel nacional y local, cuyo propósito fue el de aliviar las tensiones generadas en lo glocal por efecto de las políticas globales. Acciones que se vieron reflejadas en proyectos de investigación para la reconversión productiva.

En los años 90, se elige el primer alcalde en Puerres por voto popular, hay percepción de debilidad institucional para regular y reglamentar, lo que lleva al no cumplimiento de leyes, políticas agropecuarias con bajo impacto en la región. A finales del siglo anterior, Puerres es declarado "zona roja".

A principios del siglo XXI, "...la falta de políticas sociales y agropecuarias deterioran el campo, facilitando la llegada de los ilícitos. En la segunda mitad de la década, se genera política de erradicación de la amapola, y con esto se hace inversión social y programas de capacitación. Asimismo, se da la compra de votos por tierra de reserva. Los participantes en los talleres manifiestan que, en la actualidad:

"Hay baja participación política y social, escasa veeduría por parte de la comunidad, voto por interés, priman los intereses particulares, los recursos de programas y proyectos no llegan al campo. Hay prioridad de los temas urbanos sobre los temas del campo".

\subsection{Mapas de recursos y uso de la tierra}

Para evidenciar la percepción de los pobladores rurales del municipio de Puerres frente a los recursos del territorio y el uso de la tierra, los participantes en los talleres hicieron la representación gráfica del territorio, hace 30 años y en la actualidad.

En la Figura 1 ( $\mathrm{a}$ y b) se muestra la representación gráfica del territorio efectuada por el campesinado, la que se basa en el aspecto productivo del municipio, con sus diferentes arreglos. En la grafía del territorio, efectuada por la población indígena 
(Figura 1c), el mapa del pasado se representa de acuerdo con lo mencionado por ellos:

"Aparece el área sagrada de reserva con sus lagunas y árboles nativos. El fuego además de ser considerado un elemento esencial de la vida junto con el agua, la tierra y el aire, también era el centro de unión de las familias y la comunidad, en el que se mantenía la tradición oral a través de los mitos y leyendas. Además, alrededor de él también se tomaban las decisiones en cuanto a lo productivo: ¿Cuándo era la luna llena?, ¿Cuándo se iba a sembrar? y en general se decidía el desarrollo de las actividades productivas, económicas y familiares".

En la representación actual del territorio se muestra la intensificación de la producción agropecuaria principalmente de papa y arveja. La percepción de la comunidad indígena es que, el fuego, elemento esencial para las comunidades pasadas, fue reemplazado por la tecnología, representada por las antenas y en general por las telecomunicaciones (Figura 1d).

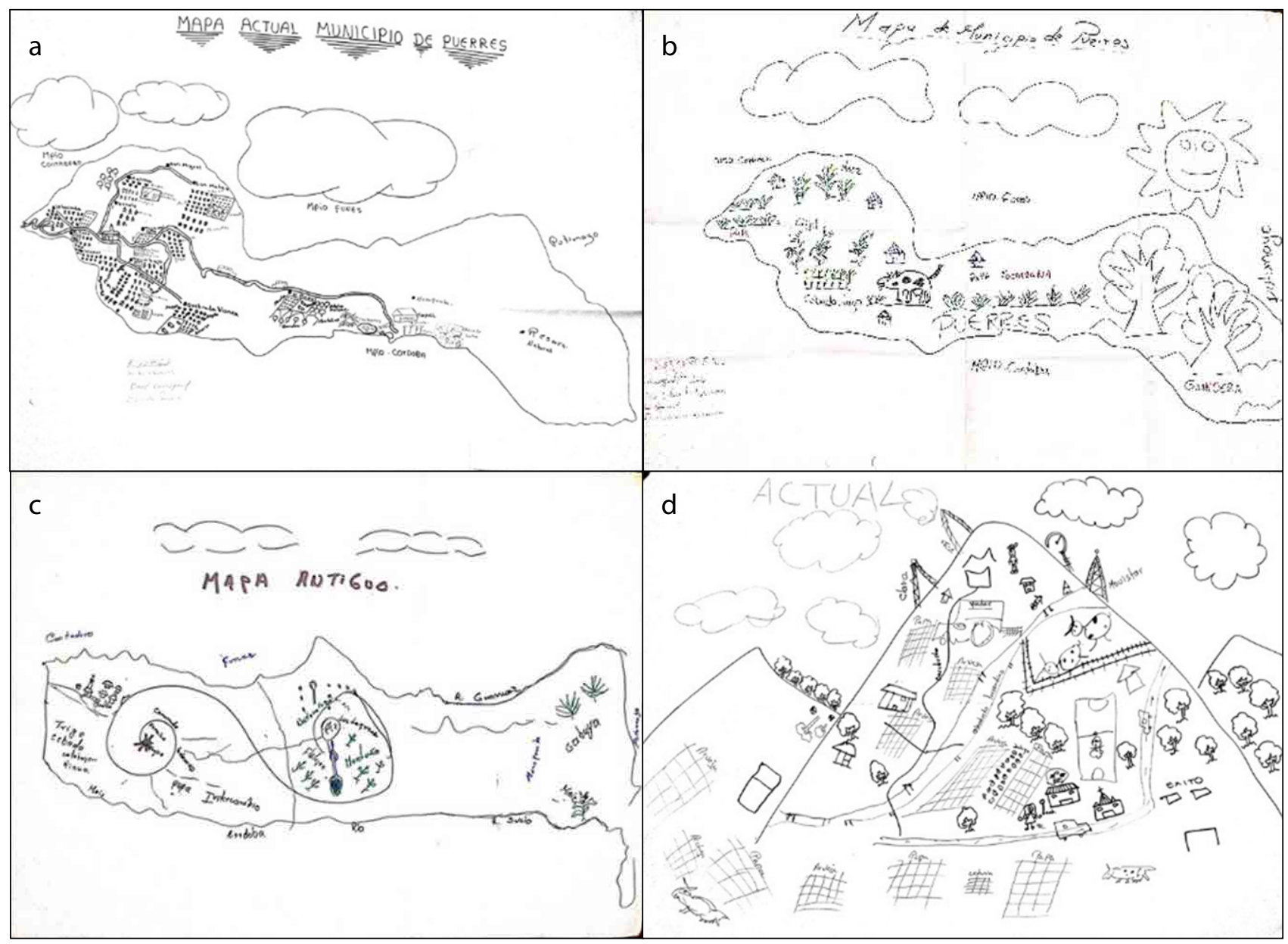

Figura 1. Mapas de recursos y uso de la tierra en Puerres: a) mapa actual, grupo de campesinado; b) mapa antiguo, grupo de campesinado; c) mapa antiguo, grupo indígena; d) mapa actual, grupo indígena. 


\subsection{Discusión}

En los ejercicios adelantados con los grupos focales de discusión, por parte de los participantes en los talleres de DRP, de manera espontánea surge la identificación de los riesgos para la seguridad del territorio, que de acuerdo con lo planteado por Wilches-Chaux (2016):

"Se entiende como la capacidad de un territorio para ofrecer a sus habitantes las condiciones de estabilidad necesarias para avanzar de manera efectiva en el aprovechamiento integral de sus capacidades; y a los ecosistemas las condiciones de estabilidad necesarias para que puedan conservar su integridad y biodiversidad y, en consecuencia, para que puedan existir y evolucionar de acuerdo con su propia naturaleza.

Dentro de los riesgos del territorio, relacionados y definidos en consenso, en términos generales, los de mayor impacto han sido los de origen antropogénico. Se identificaron tres hitos que marcaron de manera definitiva el destino del territorio, como lo fueron: i. la apertura económica de principios de los años 90, ii. constituirse en territorio de varios frentes de las FARC y iii. la llegada de la amapola hacia el año 2003. Estos acontecimientos se visibilizaron durante los talleres de DRP, como determinantes en la reconfiguración del territorio, junto con cambios vertiginosos en todas las dinámicas del ecosistema. Esta situación generó la percepción de inseguridad y de abandono por parte del Estado, así como la pérdida de gobernanza.

Lo anterior se encuentra en línea con el Informe Nacional de Desarrollo Humano, INDH 2011:

(...) Colombia rural se caracteriza por procesos de modernización apuntalados en un orden social injusto que lleva al fracaso del modelo de desarroIlo...la profundización de los conflictos, el arraigo del narcotráfico, la falta de voluntad política del Estado para contener este desorden y la continuidad de la acción de los grupos armados ilegales y legales. (INDH, 2011, citado por Salgado, 2016, p. 65).
De acuerdo con Wilches-Chaux (2016), las interacciones permanentes entre dinámicas ecosistémicas y las dinámicas de las comunidades, dan origen a un "sistema complejo" altamente sensible, en el que una causa o intervención puede generar diversos efectos favoreciendo o amenazando un territorio y sus dinámicas.

Dinámicas mundiales como: apertura económica, aumento mundial en el consumo de sustancias alucinógenas, luchas de poder y extracción de recursos, que trazaron el destino de este municipio durante un período marcado de su historia y que amenazaron de manera contundente la seguridad del territorio, generaron percepción de abandono del Estado. Por otro lado, llevó a su población a generar mecanismos de sobr evivencia que se centraron en la adaptación a las circunstancias y a los actores que confluyeron en el territorio. Desde la perspectiva de los pobladores rurales de Puerres, no gozaron de la responsabilidad, ni compensación de acciones globales que incidieran en lo glocal.

En lo productivo, el mecanismo de sobrevivencia consistió en la migración a otros sistemas de producción, como: arveja, papa, ganado para la producción de leche y frutales, sistemas que 30 o 40 años atrás no representaban la fuerza productiva del municipio, pero que llegaron a constituirse en el sustento de muy buena parte de la población. Es así como algunas especies cultivadas por los ancestros (maíz, cebada, trigo, quinua, frijol, entre otros, relacionados en la tabla1), se dejaron de sembrar o se diezmaron al punto en incidir en las dinámicas sociales, culturales y económicas como lo eran el trueque y el intercambio, para alcanzar la generación de excedentes para la comercialización, y con esto dar vía al modelo capitalista. Esto trajo consigo la disminución en su uso e ingesta, lo que ha llevado a cambios en su alimentación y la consiguiente pérdida en la calidad del alimento, según lo manifiesta la comunidad.

A finales del siglo XX, llega como opción productiva la arveja tecnificada con la variedad Sindamanoy, la que hacia el año 2000 se ve seriamente afectada por 
el auge de la amapola. Esto último, profundizó aún más la reducción en productos de autoconsumo, con el fin del "aprovechamiento" de cada espacio de la parcela, al punto de sacrificar la chagra. Esto lleva a finitud la alimentación basada en productos tradicionales de sus ancestros, así como de los sistemas sociales de intercambio y trueque.

Con la llegada del programa de erradicación de cultivos ilícitos al departamento de Nariño, los campesinos e indígenas buscan alternativas productivas sostenibles económicamente, identificando la promesa de las variedades Obonuco Andina y Obonuco San Isidro, desarrolladas para municipios cerealistas del departamento. Estas variedades se constituyeron en la alternativa económica y socialmente más viable para el municipio en ese momento y que hasta ahora, según lo mencionado por Corpoica (2018), constituyen el 90\% del área cultivada de arveja en Nariño.

Las dinámicas reinantes en el territorio objeto de estudio, son el resultado de la convergencia de la inequidad, la vulneración de la población, las diferencias de género, la exclusión, la concentración de la propiedad, la vulneración de la sostenibilidad y en general, el debilitamiento de la institucionalidad, propios de enfoques de desarrollo rural pensados desde los modelos hegemónicos del país.

Se cambiaron aspectos relevantes "del buen vivir" (el cual no nace con la ley, sino que ha sido propio de las comunidades ancestrales, particularmente de las indígenas) por el de "bienestar". De acuerdo con el planteamiento de Salgado (2016), el "buen vivir" se encuentra en contravía del conjunto de valores que rigen el comportamiento individual y comunitario. Por el contrario, el "bienestar" se rige por los principios de una sociedad de consumo, que desintegra las dinámicas comunitarias y las dinámicas ecosistémicas, lo que, como lo menciona Salgado: "frustran el sentido de la vida".

La sumatoria de factores que confluyeron en el territorio, del cual forma parte el municipio de
Puerres, devela la pérdida de buen vivir. La brecha que se ha profundizado en el tiempo es percibida por el colectivo participante en los talleres de DRP, cuya acción para estrecharla, ha sido la de generar condiciones de bienestar. En este sentido, Castell e Himanen (2016), plantean que el bienestar, además de ser visto desde la perspectiva de "calidad de vida", aborda otras dimensiones humanas de seguridad personal, prevención de la violencia, protección de la dignidad, la privacidad, los derechos de comunicación y la protección contra la discriminación.

La manifestación en la comunidad del municipio de Puerres ha sido a través de la organización colectiva que se consolida con la disposición, por un lado, como cabildo indígena y por otro, la organización campesina, las cuales han venido tomando acciones para la protección de su territorio (Centro de Memoria Histórica, 2019). Si bien es evidente un proceso profundo de adaptación, en el que la comunidad se ha "sostenido" pese a las condiciones que han impactado el territorio a lo largo de casi cuatro décadas, este se encuentra lejos de ser un proceso de resiliencia social. Esto según el concepto expresado por Salgado (2018), en cuanto a que esta resiliencia es atribuida a la capacidad de autocompensación por parte de una comunidad.

\section{Conclusiones}

La suma de acontecimientos que convergieron y constriñeron de manera contundente al municipio de Puerres, en el período de estudio, derivaron en la pérdida de seguridad del territorio y dieron cabida a procesos de desterritorialización y reterritorialización.

El fraccionamiento en el tejido social de la comunidad ha llevado a la necesidad del fortalecimiento jurídico e institucional a través del reconocimiento como cabildo indígena y la formalización de asociaciones campesinas, lo que, pese a la división social, en la actualidad contribuye a tener la percepción de un territorio seguro. 
El reconocimiento de la vulneración de los derechos de los recursos del territorio, conducen a verlo como un "territorio en transición", con sostenibilidad económica, social, política y ambiental. Esto implica una visión hacia la integralidad del territorio, en donde el rol preponderante para el desarrollo rural con enfoque territorial se fundamentará en sus recursos naturales.

La confluencia de las diferentes circunstancias y agentes que disturbaron el territorio, permiten determinar que la sumatoria de estos, junto con los aspectos técnicos y la comercialización de las variedades de las arvejas Obonuco Andina y Obonuco San Isidro, sirvieron de catalizadores para su adopción efectiva. Estas variedades de arveja se constituyeron en opción productiva sostenible para la reconversión no solo de cultivos lícitos, sino además ilícitos, lo que contribuyó de manera contundente a la reconfiguración de las actuales dinámicas en el territorio.

\section{Referencias}

Casas-Herrera, J., Gil-León, J., \& Forero-Medina, M. (2018). Cambio poblacional y dinámica socioeconómica de los municipios de Boyacá. Revista de Investigación, Desarrollo e Innovación, 8 (2), 207-221. doi: https://doi.org/10.19053/20278306. v8.n2.2018.7960

Castell, M., \& Himanen, P. (2016). Capítulo I. Modelos de desarrollo en la era global de la información: Construcción de un marco analítico. En M. Castell., \& P. Himanen, Reconceptualización del desarrollo en la era global de la información, 27-46.

Castillo-Burbano, A. M., \& Jurado, J. A. (2014). Caracterización Social y Económica del Departamento de Nariño. San Juan de Pasto, Colombia.

Centro de Memoria Histórica. (enero 14 de 2019). Minga Indígena Caminando la Palabra. Marcha de todos los pueblos indígenas de Colombia. Recuperado de: http://www.centrodememoriahistorica.
gov.co/multimedias/MemoriasExpresivasRecientes/Memoria_H/cauca/minga/index.html

CEPAL. (2000). El impacto de las reformas estructurales en la agricultura colombiana.

Corporación Colombiana de Investigación Agropecuaria, CORPOICA. (S.F.). Aspectos económicos y de comercialización de arveja en Colombia y en el departamento de Nariño. Informe Económico, Nariño, Pasto.

Corporación Colombiana de Investigación Agropecuaria, CORPOICA. (2018). Balance social 2017. Bogotá, Colombia.

CORPOICA - PRONATTA. (Agosto de 2002). Obonuco Andina. Nueva variedad mejorada de arveja para el sistema de producciónde economía campesina del sur de Nariño. San Juan de Pasto, Colombia.

El Tiempo (abril 27 de 1992). Nariño: inundado de flor maldita. Recuperado de: https://www.eltiempo.com/archivo/documento/MAM-101811

Federación Nacional de Cultivadores de Cereales y Leguminosas, FENALCE. (S.F.). Resumen de Importaciones. Recuperado de: http://www.fenalce. org/nueva/plantillas/arch_down_load/Impo20102014.pdf

García-Parra, M. A., \& Plazas-Leguizamón, N. Z. (2019). Análisis del ciclo de vida de las publicaciones sobre producción de quinua (chenopdium quinua Willd), a través de curvas en S. Revista de Investigación, Desarrollo e Innovación, 9 (2), 379-391. doi: 10.19053/20278306.v9.n2.2019.9189

Gelfius, F. (2002). 80 herramientas para el desarrollo participativo: diagnóstico, planificación, monitoreo, evaluación. San José de Costa Rica: IICA.

ICA-CORPOICA. (1995). ICA-CORPOICA Sindamanoy. Variedad mejorada de arveja para clima frío. Pasto, Colombia. Recuperado de: 
https://repository.agrosavia.co/bitstream/handle/20.500.12324/20575/42004_45065.pdf?sequence $=1$ \&isAllowed $=y$

Martínez-Miguélez, M. (2004). Ciencia y arte en la metodología Cualitativa. México: Trillas.

Ministerio de Agricultura y Desarrollo Rural. (2014). Arveja. Evaluaciones Agropecuarias Municipales. Recuperado de: http://www.agronet.gov.co/Documents/Arveja.pdf

Molina, C, Á. J. (2009). Identificación de factores incidentes en las decisiones de adopción de tecnología en productores ganaderos criadores familiares. Agrociencia, XIII (2), 70-83. Recuperado de: http://www.fagro.edu.uy/agrociencia/index.php/ directorio/article/view/152

Organización para la Cooperación y el Desarrollo Económico, OCDE. (2013). Estudios de la OCDE de las políticas de innovación: Colombia. Evaluación General y recomendaciones.

Salgado, C. (2016). Colombia: Estado actual del debate sobre el desarrollo rural. Bogotá, Colombia: Desde abajo.

Salgado, C. (marzo 2 de 2018). Notas en Seminario de Síntesis Maestría en Desarrollo Rural. Universidad Pedagógica y Tecnológica de Colombia, Tunja, Colombia.
Silva, E. L., \& Menezes, E. M. (2005). Metodologia da Pesquisa e Elaboração de Dissertação. Florianópolis, Brasil:UFSC.

Valderrama-Hernández, R. (2013). Diagnóstico participativo con cartografía social. Innovaciones en metodologías Investigación-Participativa (IAP). Revista Andaluza de Ciencias Sociales, 12, 53-65. Recuperado de: https://revistascientificas.us.es/ index.php/anduli/article/view/3635

Vásquez, A. (2007). Desarrollo endógeno. Teorías y Políticas de desarrollo territorial. Investigaciones Regionales - Panorama y Debates, 11, 183210. Recuperado de: https://www.redalyc.org/ pdf/289/28901109.pdf

Wilches-Chaux, G. (2016). Base ambiental para la paz. La necesidad de hacerle gestión del riesgo al paz-conflicto. En O. N. Paz, Colección Estudios Técnicos Para la Construcción de la Paz, 42. Bogotá: Planeta Paz.

Zuluaga-Mogollón, M. (2019). Adopción tecnológica como estrategia de Desarrollo Local: El caso de las variedades de arveja (Pisum sativum) obonuco Andina y Obonuco san Isidro en los municipios de Córdoba y Puerres, Nariño (Colombia) 2000-2015 (Tesis de maestría). Universidad Pedagógica y Tecnológica de Colombia, Tunja, Colombia. 
